\title{
Internal image potential in semiconductors: Effect on scanning tunneling microscopy
}

\author{
Z.-H. Huang, M. Weimer, and R. E. Allen \\ Department of Physics, Texas A\&M University, College Station, Texas 77843-4242
}

(Received 26 July 1993)

\begin{abstract}
The tunneling of electrons from a semiconductor surface to a metal tip, across a vacuum gap, is influenced by two image interactions: an attractive image potential in the vacuum region, which lowers the apparent tunneling barrier, and a repulsive image potential in the semiconductor interior, which raises it for conduction-band electrons. We report on detailed calculations of tunneling currents and apparent barrier heights for a model metal-vacuum-semiconductor junction which utilize semiclassical dielectric functions to compute the image potential in all three regions. The effect of image forces is found to be small compared to that of either the vacuum barrier or tip-induced band bending. In particular, the image-induced barrier in the semiconductor has only a minor influence on either the apparent barrier height or the shape of current-voltage characteristics, both of which are routinely measured in scanning-tunneling-microscopy experiments. This finding is explained by a qualitative WKB analysis and several simple arguments.
\end{abstract}

\section{INTRODUCTION}

Electron tunneling through a junction is often treated by considering independent electrons moving in an effective potential. The simplest model potential for a metal-vacuum-metal (MVM) junction is the rectangular barrier, but it is well known that image forces modify this potential significantly. Calculations based on classical electrostatics, for example, reveal that the image interaction reduces the effective vacuum barrier by lowering its maximum height and slightly narrowing its width. ${ }^{1-3}$ More realistic quantum-mechanical calculations employing the density-functional formalism indicate that the lowest-order correction to this classical picture is simply a displacement of the effective image plane by several tenths of an angstrom, along with a smooth matching of the vacuum potential outside the surface to the minimum of the conduction band in the metal interior. ${ }^{4,5}$ The resulting vacuum barrier height then depends on the gap spacing $s$, and is well approximated by ${ }^{6}$

$$
\Phi(s)=\Phi_{0}-\frac{\alpha}{s-s_{0}},
$$

where $\Phi_{0}$ represents the average work function of the metal electrodes, $s_{0}$ accounts for the shift in image plane positions, and $\alpha \sim 10 \mathrm{eV} \AA$.

The scanning tunneling microscope (STM), with which one can precisely adjust $s$, and hence continuously vary the vacuum barrier, is a potentially valuable tool for investigating this effect. By modulating the distance between tip and sample at a frequency outside the microscope's feedback bandwidth, and detecting a synchronous ac tunnel current, one can extract an apparent barrier height ${ }^{7}$ defined by

$$
\Phi_{A}=[(-1 / A) d \ln I / d s]^{2},
$$

where $A=1.025 \mathrm{eV}^{-1 / 2} \AA^{-1}$. The utility of (2) stems both from the fact that it is an experimentally accessible quantity, and that in a Wentzel-Kramers-Brillouin (WKB) approximation for the rectangular barrier it yields the correct barrier height. Early experiments on metals, however, revealed no discernible reduction in $\Phi_{A}$ by image forces at separations of more than a few angstroms. This insensitivity is due not to the absence of image effects, but, rather, to the particular form of the potential in (1), for which a WKB analysis shows that the first-order term in an expansion of $\Phi_{A}$ in powers of $s^{-1}$ vanishes. ${ }^{6}$ Similar lines of reasoning further supported the conclusion that the apparent barrier height (2) is unlikely to be a sensitive probe of the image potential in MVM tunneling at large distances. ${ }^{8}$ Nevertheless, the question of image effects and their relevance to STM experiments continues to hold great interest. Subsequent investigators have addressed a number of fundamental issues, including the role of nonplanar geometry, ${ }^{9-12}$ the precise nature of the effective vacuum barrier at short distances, ${ }^{7,13,14}$ and the influence of the dynamic image interaction in time-dependent tunneling. ${ }^{15}$

If we replace one of the metal electrodes by a semiconductor to form a metal-vacuum-semiconductor (MVS) junction, then the image correction to the vacuum potential at large distances will be similar to that of the MVM junction when scaled to reflect the dielectric behavior of the semiconductor. ${ }^{16}$ A qualitatively different phenomenon now appears, however, in that tunneling electrons experience an additional image force in the semiconductor interior due to the finite polarizability of that medium. In a classical multiple-image analysis of the planar MVS or semiconductor-vacuumsemiconductor (SVS) junction, this "internal" image potential is given by the expression ${ }^{17,18}$

$$
V_{s}(z)=\frac{q^{2}}{16 \pi \epsilon_{s}} \sum_{n=0}^{\infty}\left(\beta \beta^{\prime}\right)^{n}\left[\frac{\beta}{n s-z}-\frac{\beta^{\prime}}{(n+1) s-z}\right] \text {, }
$$

for an extra point charge $q$ located at $z<0$, with $z=0$ the position of the semiconductor-vacuum interface, and 
$z=s$ the position of the vacuum-metal interface. Here, $\epsilon_{s}$ is the static dielectric permittivity of the semiconductor and

$$
\beta=\frac{\epsilon_{s}-\epsilon_{0}}{\epsilon_{s}+\epsilon_{0}},
$$

where $\epsilon_{0}$ is the permittivity of vacuum; a corresponding expression defines $\beta^{\prime}$. For an MVS junction, $\beta^{\prime}=1$. The internal image potential (3) leads to an effective increase in the semiconductor band gap near an ideal semiconductor-vacuum interface, since electrons in the conduction band are repelled from the surface whereas electrons in the valence band are attracted. ${ }^{17}$ Imageinduced features of this kind have, in fact, recently been proposed as an explanation for the thickness-dependent energy shifts observed in both $\mathrm{x}$-ray photoemission spectroscopy and Auger electron spectroscopy spectra of thin $\mathrm{SiO}_{2}$ films on silicon substrates. ${ }^{19}$ The situation is exactly reversed at an ideal semiconductor-metal interface where the band gap is effectively decreased, a phenomenon already anticipated from the quantum many-body point of view. $^{20}$

To explicitly show the functional dependence on gap spacing, Eq. (3) may be written in scaled form ${ }^{18}$ as

$$
V_{s}(z)=\frac{q^{2}}{8 \pi \epsilon_{0} S} U_{s}\left(\beta, \beta^{\prime}, \xi\right),
$$

where $\xi=z / s$ and $U_{s}$ is a dimensionless function independent of $s$. In an MVS junction, $V_{s}(z)$ changes sign at a position $z_{0}=s \xi_{0}(\beta)$ because the contribution from polarization charge at the metal-vacuum interface eventually dominates that of the semiconductor-vacuum interface deep in the semiconductor interior. Thus, a conductionband electron approaching the surface will experience an image interaction which is at first attractive, and then repulsive, with

$$
\begin{array}{ll}
U_{s}<0, & \xi<\xi_{0}(\beta) \\
U_{s}=0, & \xi=\xi_{0}(\beta) \\
U_{s}>0, & \xi>\xi_{0}(\beta) .
\end{array}
$$

The image-induced tunneling barrier in the semiconductor is then strongly dependent on electrode separation. It is appealing, therefore, to examine the possibility of detecting this additional barrier using the scanning tunneling microscope.

The classical expression for the image potential is inconvenient to use in tunneling calculations implementing transfer matrix methods, since it diverges at the interfaces, where unphysical bound states may be introduced. A semiclassical theory for the MVS junction which removes these divergences, but nevertheless requires a smooth interpolation for the total effective potential in the near interfacial region, has recently been discussed. ${ }^{21}$ Initial calculations based on this theory showed that, for the special case of vanishing electric field between tip and sample, the contribution to $\Phi_{A}$ from the image-induced barrier in the semiconductor is small. The insensitivity of $\Phi_{A}$ to this additional correction, it was argued, could be ascribed to the short-range nature of the induced barrier in the semiconductor, which therefore appears nearly transparent. It is the purpose of this paper to reexamine the question of observing such image effects in an MVS junction under more general circumstances.

To do so, we first review briefly the phenomenology of apparent barrier heights in tunneling experiments at unpinned semiconductor surfaces. At zero applied bias there will, in general, be a space charge induced in the semiconductor by the difference between tip and sample work functions, $\Delta \Phi=\Phi-\left(\chi+\phi_{n}\right)$, as shown in Fig. 1(a) for $n$-type material in depletion. This space charge may be eliminated only by applying an appropriate bias voltage, $V_{\mathrm{FB}}=-\Delta \Phi$, to recover the flat-band condition illustrated in Fig. 1(b). Tip-induced band bending of the kind indicated in Fig. 1(a) will dramatically affect STM measurements of $\Phi_{A}$, for reasons which are easy to appreciate. ${ }^{22}$ The surface potential $V_{d}$ is a monotonically decreasing function of $s$, with a maximum at $s=0$. Since the depletion width $W$ is typically much longer than $s$, only those carriers with a thermally activated kinetic energy greater than $V_{d}$ will surmount the diffusion barrier to arrive at the semiconductor-vacuum interface. In-
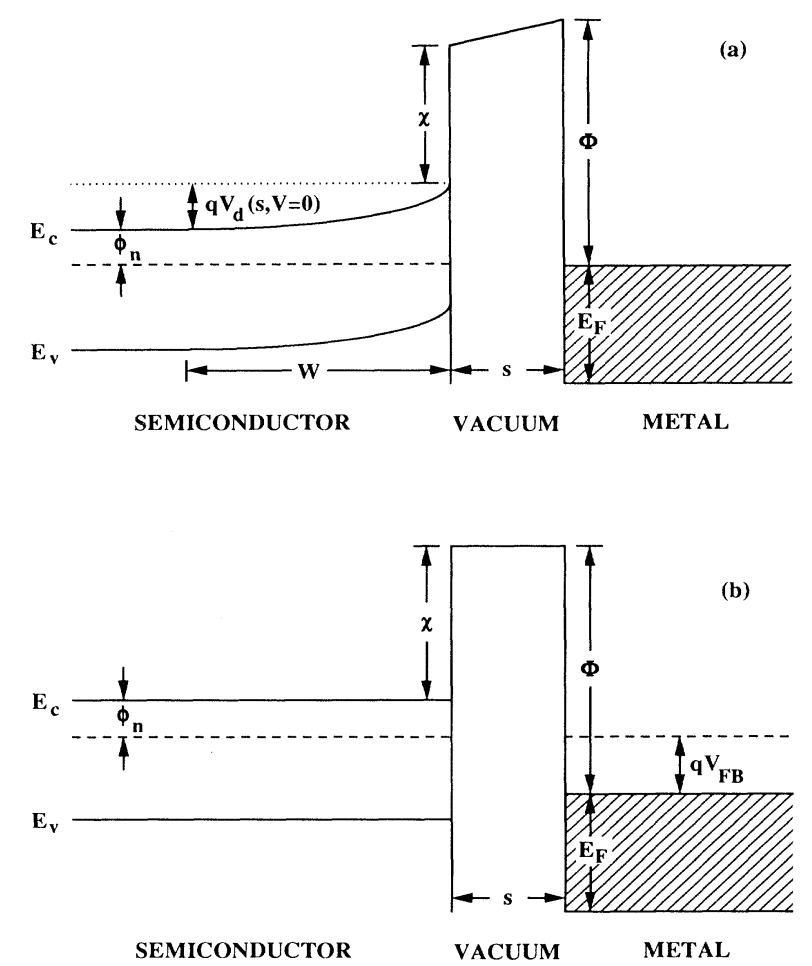

FIG. 1. (a) Schematic energy diagram for an ideal semiconductor-vacuum-metal junction at zero applied bias $V$, neglecting image effects. $\Phi$ is the metal work function, $E_{F}$ the metal Fermi energy, $\chi$ the semiconductor electron affinity, and $\phi_{n}$ the offset from the Fermi level to the bottom of the semiconductor conduction band. The drawing is not to scale since the depletion width $W$ is typically much greater than the gap spacing $s$, and $\chi$ exceeds the band gap, $E_{c}-E_{v}$. (b) Flatband situation when the applied bias $V_{\mathrm{FB}}$ has been chosen to eliminate the electric field arising from a difference in metal and semiconductor work functions, $\Phi-\left(\chi+\phi_{n}\right)$. 
creasing the separation between tip and sample exponentially suppresses tunneling through the vacuum barrier as before, but now also exponentially enhances the number of electrons reaching the semiconductor surface. Because of these two competing factors, the observed variation in current density is less than expected; this is then interpreted as a smaller barrier height in Eq. (2). If the image-induced potential (5) is superimposed on the space-charge potential of Fig. 1(a), it too will change with gap spacing. Since the energy scale characterizing the carrier distribution is $k T$, we may now ask whether inclusion of the semiconductor image potential further perturbs the carrier distribution, and thus the logarithmic derivative of the tunneling current, in any measurable way.

In what follows, we first present key elements of the semiclassical image potential theory, and describe our method of calculation, in Sec. II. Predictions for the apparent barrier height and tunneling current-voltage characteristics, including image effects, are presented in Sec. III for the particular case of a planar, $n$-type hydrogen-passivated $\mathrm{Si}(111) / \mathrm{Au}$ junction. We devote Sec. IV to a qualitative discussion of these results within the framework of a WKB analysis, which permits us to generalize our findings beyond the specific materials we have chosen to study in detail. Section V summarizes our conclusions.

\section{DESCRIPTION OF THE CALCULATION}

Our calculations are carried out under the assumption that the system consists of independent electrons moving in an effective one-dimensional potential. The bulk metal is treated as a free-electron Fermi gas characterized by Fermi energy $E_{F}$ and work function $\Phi$. The semiconductor is modeled in a one-band effective-mass approximation including anisotropy. When assembled into an MVS junction, the semiconductor-vacuum and vacuum-metal interfaces perturb these bulk effective potentials; the corrections we specifically consider here are introduced by the multiple-image interaction and tip-induced space charges. Modifications due to surface states or collective surface excitations are ignored.

The expressions for the classical image potential in an ideal metal-insulator-semiconductor (MIS) junction similar to that shown in Fig. 1 diverge at the semiconductorvacuum and vacuum-metal interfaces. ${ }^{17,18}$ These unphysical divergences may be circumvented by employing an effective Thomas-Fermi theory of dielectric screening ${ }^{23,24}$ to derive a semiclassical expression for the image potential experienced by a tunneling electron. Specifically, the Thomas-Fermi dielectric function in a bulk metal is given by $\epsilon_{m}(k) / \epsilon_{0}=1+\gamma_{m}^{2} / k^{2}$, where $\gamma_{m}$ is the corresponding Thomas-Fermi wave vector. Within the same approximation, the screening response of an intrinsic bulk semiconductor may be modeled by the dielectric function ${ }^{23}$

$$
\frac{\epsilon_{s}(k)}{\epsilon_{0}}=1+\frac{\left(\epsilon_{s} / \epsilon_{0}\right)-1}{1+\left(\epsilon_{s} / \epsilon_{0}\right)\left(k^{2} / \gamma_{s}^{2}\right)},
$$

where $\left(\epsilon_{s} / \epsilon_{0}\right)$ is the long-wavelength static dielectric constant and $\gamma_{s}$ an effective Thomas-Fermi wave vector which depends on the total valence charge density. It can then be shown ${ }^{21}$ that the resulting semiclassical expression for the image potential experienced by a point charge $q$ located at position $z<0$ in the semiconductor is

$V_{s}(z)=\frac{q^{2}}{8 \pi \epsilon_{s}} \int_{0}^{\infty}\left\{F_{k}^{s} e^{k z}+A_{k}^{s} \exp \left[\left(k^{2}+\gamma_{s}^{2}\right)^{1 / 2} z\right]\right\} d k$,

where

$$
F_{k}^{s}=-\frac{e^{-2 k s}-\beta}{1-\beta e^{-2 k s}} e^{k z},
$$

coincides with the classical solution ${ }^{17,18}$ and

$$
A_{k}^{s}=-\frac{\left\{\frac{2 \alpha_{s}}{1+\alpha_{s}}\right\}\left(\eta_{m} e^{-k(2 s-z)}+F_{k}^{s}\right)+\left\{\frac{2 \beta}{1-\beta}\right\}\left(\eta_{m} e^{-2 k s}-\eta_{s}\right) \exp \left[\left(k^{2}+\gamma_{s}^{2}\right)^{1 / 2} z\right]}{1-\eta_{s} \eta_{m} e^{-2 k s}},
$$

represents an additional response arising from shortrange screening in the semiconductor. Here, we define, in addition to $\beta$ in (4) above,

$$
\begin{aligned}
& \alpha_{m}(k)=\frac{k}{\left(k^{2}+\gamma_{m}^{2}\right)^{1 / 2}}, \\
& \alpha_{s}(k)=\frac{k}{\left(k^{2}+\gamma_{s}^{2}\right)^{1 / 2}}, \\
& \eta_{m}(k)=\frac{1-\alpha_{m}(k)}{1+\alpha_{m}(k)}, \\
& \eta_{s}(k)=\frac{1-\alpha_{s}(k)}{1+\alpha_{s}(k)} .
\end{aligned}
$$

When $q$ is located in the vacuum region $0<z<s$, the corresponding expression for the semiclassical image potential becomes

$$
V_{v}(z)=\frac{q^{2}}{8 \pi \epsilon_{0}} \int_{0}^{\infty}\left(B_{k}^{v} e^{k z}+C_{k}^{v} e^{-k z}\right) d k,
$$

where

$B_{k}^{v}=-\eta_{m} e^{-k s} \frac{\left\{\frac{1-\beta}{1+\beta}\right\} \eta_{s} F_{k}^{v}-\eta_{s} e^{k(s-z)}+e^{k(s+z)}}{e^{2 k s}-\eta_{s} \eta_{m}}$, 
$C_{k}^{v}=\eta_{s} e^{k s} \frac{\left\{\frac{1-\beta}{1+\beta}\right\} F_{k}^{v}-e^{k(s-z)}+\eta_{m} e^{-k(s-z)}}{e^{2 k s}-\eta_{s} \eta_{m}}$,

and

$$
F_{k}^{v}=(1+\beta) \frac{e^{k(s-z)}-e^{-k(s-z)}}{1-\beta e^{-2 k s}} .
$$

Finally, for $q$ in the metal $(z>s)$ one finds

$V_{m}(z)=\frac{q^{2}}{8 \pi \epsilon_{0}} \int_{0}^{\infty} \exp \left[\left(k^{2}+\gamma_{m}^{2}\right)^{1 / 2}(s-z)\right] D_{k}^{m} d k$,

with

$$
D_{k}^{m}=\frac{\eta_{m}-\eta_{s} e^{-2 k s}}{1-\eta_{s} \eta_{m} e^{-2 k s}} \exp \left[\left(k^{2}+\gamma_{m}^{2}\right)^{1 / 2}(s-z)\right]
$$

representing the effect of short-range screening. It is easily verified that the formulas for $V_{s}(z), V_{v}(z)$, and $V_{m}(z)$ given above reproduce the expected classical results in the limits $\gamma_{s, m} \rightarrow \infty$, and that $V_{m}(z)$, in particular, vanishes.

An effective one-electron potential is obtained throughout the junction by adding the appropriate regional solution for the semiclassical image potential to the band diagram shown in Fig. 1. We first consider the case illustrated in Fig. 1(b) when the applied bias $V_{\text {FB }}$ has been chosen to eliminate any electric field between tip and sample in the absence of image corrections. Selecting the bottom of the semiconductor conduction band as an energy reference, the one-electron potential is then given by $V_{s}(z)$ in the semiconductor, $V_{v}(z)+\chi$ in the vacuum gap, and $V_{m}(z)+\chi-\left(\Phi+E_{F}\right)$ in the metal. This function, though finite, is still discontinuous at the semiconductor-vacuum and vacuum-metal boundaries. There are a number of reasons for this discontinuity, which originates from inadequacies in the semiclassical treatment at distances within a screening length of the interfaces. First, the treatment assumes bulk values for the screening parameters in each medium. Second, the correct behavior of the total effective potential (or selfenergy) experienced by an electron moving from inside a polarizable medium to the region immediately outside it, can only be deduced by taking full account of many-body effects. ${ }^{4,5}$ Finally, there is no a priori relationship between the effective band bending produced by image effects in the immediate vicinity of an interface, and the one-electron potential used to model band structure, which guarantees that the contribution from surface dipole layers, together with exchange and correlation effects, will produce a smoothly varying total potential across the interfaces. Since the true one-electron potential is presumably continuous when all of these effects are self-consistently accounted for, we supplement the semiclassical picture by employing a third-order polynomial to smoothly join the calculated potential and its derivative at points a distance $\gamma_{s, m}^{-1}$ on either side of the semiconductor-vacuum and vacuum-metal boundaries. This interpolation is then used to represent the tunneling potential in the near-interfacial regions. An example of this interpolated potential is presented in Fig. 2(a) for a junction consisting of a $n$-type hydrogen-terminated $\mathrm{Si}$ (111) surface and a gold tip electrode [with $\gamma_{m}=1.7$ $\AA^{-1}, \gamma_{s}=1.16 \AA^{-1}$, and $\epsilon_{s}=11.9$ (Refs. 25-27)], where we contrast our results with the classical calculation.

The more general situation occurs when $V \neq V_{\mathrm{FB}}$, and there is a nonvanishing field in the vacuum region. In the absence of surface states this field penetrates the semiconductor interior, and the resultant band bending due to the tip-induced space charge is schematically illustrated in Fig. 1(a) for $n$-type material when $\Delta \Phi=\Phi-\left(\chi+\phi_{n}\right)>0$. The surface potential $q V_{d}$ is a sensitive function of both gap spacing and bias voltage, and is given in the depletion approximation by $^{22}$
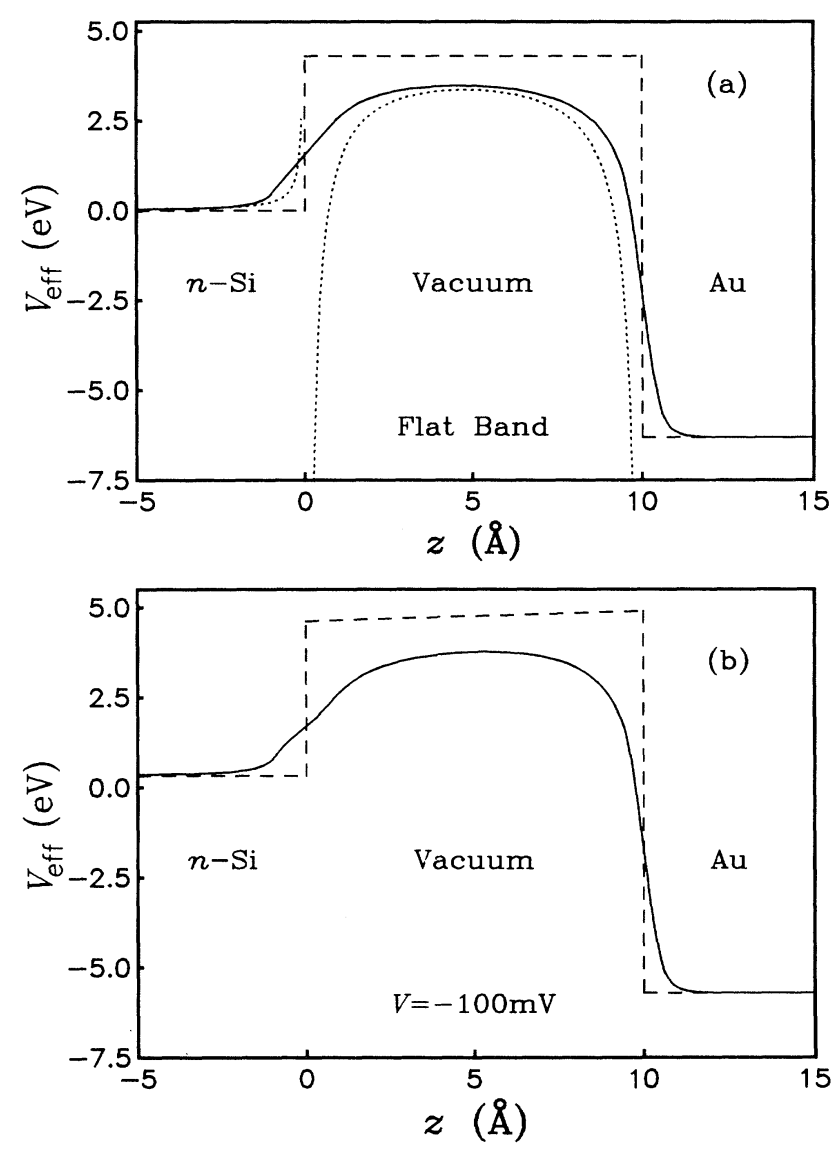

FIG. 2. (a) Effective one-electron potential energy as a function of position for $V=V_{\mathrm{FB}}$ and $s=10 \AA$. Solid line: including the semiclassical image potential with interpolation. Dotted line: including the classical image interaction. Dashed line: neglecting image effects. $\chi=4.3 \mathrm{eV}, \Phi=5.1 \mathrm{eV}$, and $E_{F}=5.53$ $\mathrm{eV}$. The potentials are independent of doping. (b) Effective one-electron potential energy as a function of position at a representative bias which produces depletion in the semiconductor $\left(N_{d}=5 \times 10^{17} \mathrm{~cm}^{-3}\right)$. All other parameters as above. Solid line: including the semiclassical image potential with interpolation. Dashed line: neglecting image effects. Note the offset of the semiconductor surface potential from the conduction band edge in the bulk. 


$$
q V_{d}(s, V)=\Delta \Phi\left\{\left[1+\left(s / s_{0}\right)^{2}+q V / \Delta \Phi\right]^{1 / 2}-s / s_{0}\right\}^{2} .
$$

The resulting electrostatic potential in the semiconductor interior, $\psi(z)$, has finite range, vanishing at $z=-W$, and follows the well-known form ${ }^{27}$

$$
\psi(z)=V_{d}(1+z / W)^{2},
$$

for $-W<z<0$. The depletion width $W$ is a function of both the bulk donor density $N_{d}$ and the semiconductor surface potential $q V_{d}$ through $^{27}$

$$
W\left(q V_{d}\right)=\left(2 \epsilon_{s} V_{d} / q N_{d}\right)^{1 / 2},
$$

while the length scale $s_{0}$ is obtained from the zero-bias, zero-separation depletion width according to $^{22}$

$$
s_{0}=\left(\epsilon_{0} / \epsilon_{s}\right) W(\Delta \Phi) \text {. }
$$

The total one-electron potential now includes a contribution from $\psi(z)$ as well as that from $V_{s}(z)$ for $z<0$. As above, we interpolate within the near-interfacial region to obtain a continuous result as a function of position, and an example calculated for the same hydrogen-terminated $\mathrm{Si}(111) / \mathrm{Au}$ junction, at a sample bias of $-100 \mathrm{mV}$ with $N_{d}=5 \times 10^{17} \mathrm{~cm}^{-3}$, is illustrated in Fig. $2(\mathrm{~b})$, where the additional long-range effect of Debye screening due to free carriers has been ignored.

To compute a tunneling current density, the appropriate potential is substituted into a one-dimensional effective-mass Schrödinger equation for electrons originating from the conduction band of the semiconductor. This equation is then solved numerically to evaluate a transmission probability $D\left(E_{z}, s\right)$, where $E_{z}$ is the energy component normal to the barrier. The net tunneling current density is given by the expression

$$
j(s)=\frac{e m_{t}}{\gamma^{1 / 2} m_{0}} \int_{0}^{\infty} d E_{z} D\left(E_{z}, s\right)\left[N_{0}\left(E_{z}\right)-N_{0}\left(E_{z}-q V\right)\right],
$$

where $m_{0}$ is the free-electron mass, $N_{0}\left(E_{z}\right)$ and $N_{0}\left(E_{z}-q V\right)$ are one-dimensional finite-temperature supply functions ${ }^{28}$ for electrons in the semiconductor and metal, respectively, and $\gamma$ represents an anisotropy factor for the constant-energy surfaces of the equivalent $\mathrm{Si}\langle 100\rangle$ ellipsoidal pockets at the indirect conductionband minimum projected along the (111) direction. The resulting effective mass for tunneling in the (111) direction is $m_{z}^{*}=\gamma m_{l}=0.26 m_{0}$. Further details concerning the calculation of $D\left(E_{z}, s\right)$ may be found in Ref. 22 .

\section{RESULTS}

The tunneling current density as a function of gap spacing calculated on the basis of Eq. (25), using the oneelectron effective potential of Fig. 2(b), is presented in Fig. 3 for a representative bias voltage which produces substantial majority carrier depletion in the near surface region of the semiconductor. For comparison, the results obtained using the potential of Fig. 1(a), neglecting image effects, are also shown. At each of the doping levels con- sidered, we see roughly an order of magnitude increase in the overall current density at any reasonable gap separation due to the inclusion of image forces. The shape of the $j-s$ curves on a semilogarithmic plot deviates at small $s$ from the straight line predicted for vacuum tunneling because of the influence of tip-induced space charge on current transport through the junction, as explained previously. ${ }^{22}$ What we wish to call attention to here is that the $j-s$ characteristics for a given doping display a similar shape whether or not image effects are included.

This observation is more quantitatively expressed in Fig. 4, where an apparent barrier height as a function of gap spacing is obtained via Eq. (2) from the curves in Fig. 3. We see that the barrier height displays nearly the same behavior as a function of $s$ (for a given doping) with or without image forces, but that including image effects results in an overall lowering of the apparent barrier by a few tenths of an eV. Also presented in Fig. 4 are the doping-independent results for $\Phi_{A}$ when the applied bias has been chosen to eliminate any tip-induced band bending, ${ }^{21}$ as shown in Fig. 1(b). It is clear from the figure that the principal influence on $\Phi_{A}$ arises from tipinduced space charge, and that the image potential constitutes a relatively small perturbation.

The influence of image forces on the tunneling current-voltage characteristics at constant separation may be calculated in a manner similar to the $j-s$ characteristics presented above, and the results are shown in

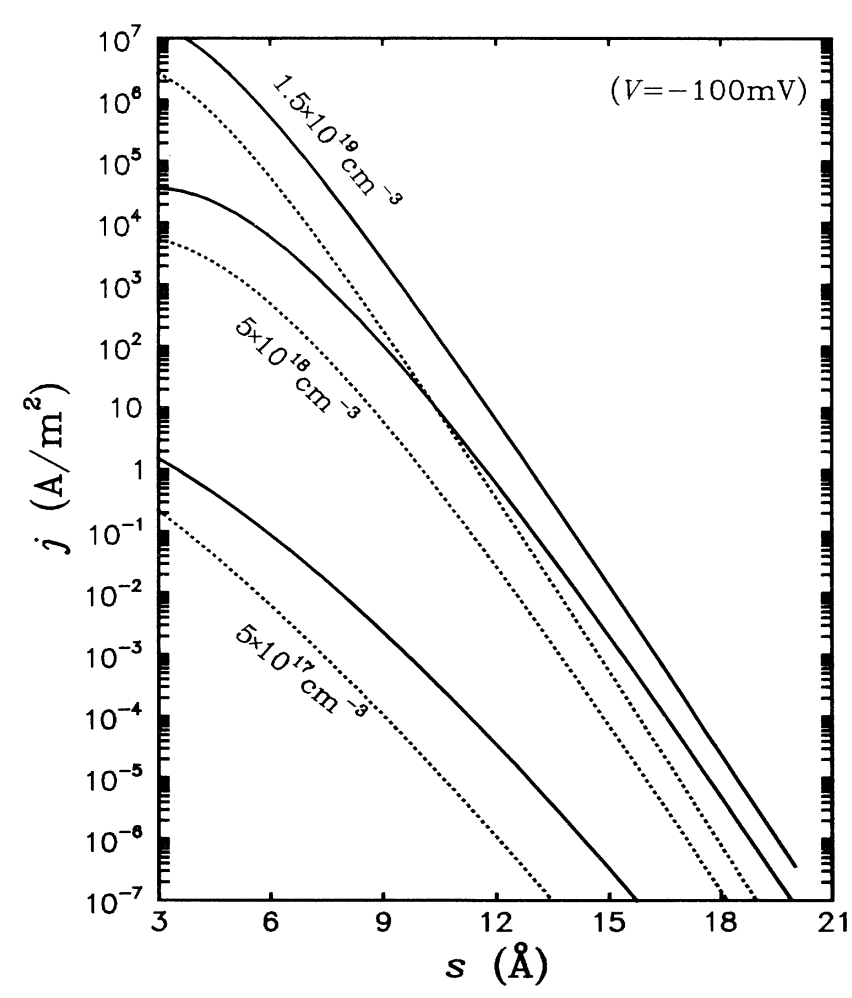

FIG. 3. Tunneling current density $j$ versus electrode separation $s$ as a function of doping for fixed bias and $T=300 \mathrm{~K}$. Solid line: including the semiclassical image potential as in Fig. 2(b). Dashed line: neglecting image effects. 


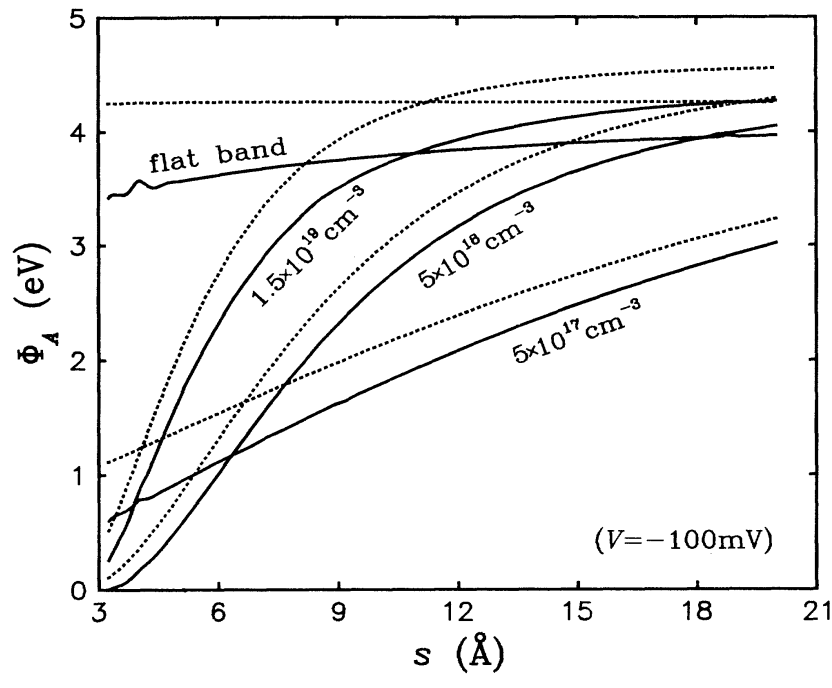

FIG. 4. Apparent barrier height $\Phi_{A}$ versus electrode separation $s$ as a function of doping. Solid line: including the semiclassical image potential. Dashed line: neglecting image effects. The flatband results are essentially independent of doping in the nondegenerate regime.

Fig. 5, as a function of doping, for $s=5 \AA$. To facilitate a comparison between $j-V$ curves calculated with and without image corrections at fixed doping, and between $j-V$ curves obtained for different doping levels, both the current density and applied bias have been scaled to their respective flatband values. As already noted, the image potential increases the current density for a given applied bias and electrode separation, but we see here that the overall shape of the $j$ - $V$ characteristic remains essentially

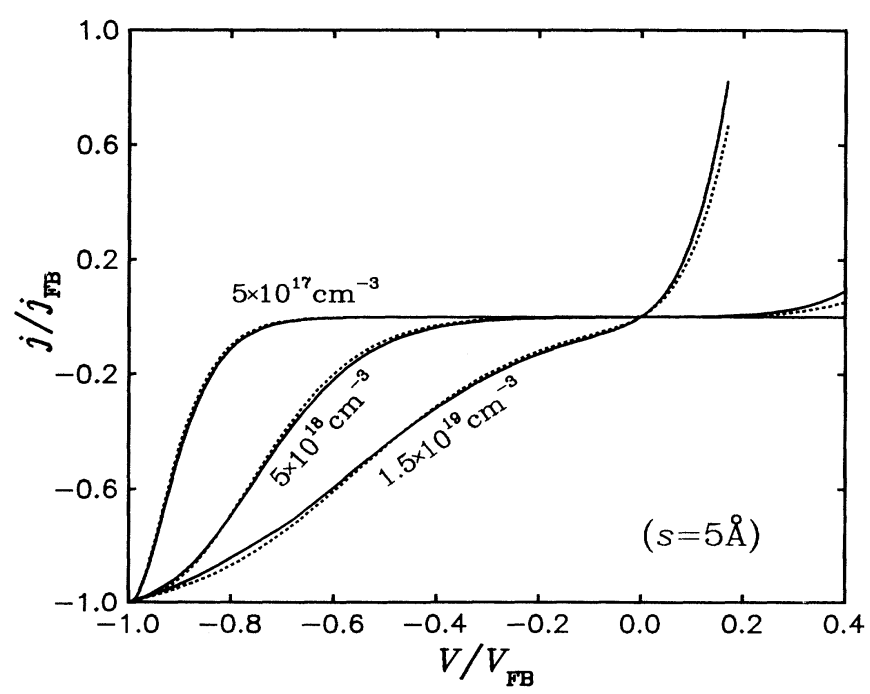

FIG. 5. Tunneling current density $j$ versus applied bias $V$ as a function of doping for fixed separation and $T=300 \mathrm{~K}$. Solid line: including the semiclassical image potential. Dashed line: neglecting image effects. The current density and applied bias are scaled to their respective flatband values to facilitate a comparison of the shape of the $j-V$ characteristics. unchanged. This shape is, again, dominated by the tipinduced space charge. A discussion of the somewhat unusual properties of the reverse-bias characteristics may be found in the literature. ${ }^{29,30}$

\section{DISCUSSION}

Our results for the specific case of a planar, passivated $n$-type $\mathrm{Si}(111) / \mathrm{Au}$ junction indicate that the influence of image effects on the apparent barrier height is small, and may not be experimentally detectable in practice. The role of tip-induced space charge in determining the apparent barrier decreases with increasing bias as flatband conditions are approached, ${ }^{22}$ so that image effects, if detectable, will be most prominent at bias voltages very near $V_{\mathrm{FB}}$. The shape of the tunneling current-voltage characteristics also appears to be insensitive to image forces.

In what follows, we examine these conclusions from the perspective of a WKB analysis of tunneling through the compound barrier created either by the space charge in the semiconductor neglecting image effects, followed by the vacuum gap, or the image potential in the semiconductor neglecting space charge, again followed by the vacuum gap. In this way we can identify important qualitative features of the realistic junction problem containing both elements which will give us some insight into the relevance of specific material parameters to our results, and the circumstances under which image effects might play a more significant role.

The simplest case arises when the only barrier that need be considered is due to the vacuum gap. If we denote by $D^{\mathrm{vac}}\left(E_{z}, s\right)$ the energy-dependent transmission coefficient, then in the WKB approximation one obtains

$$
\ln D^{\mathrm{vac}}\left(E_{z}, s\right)=-2\left(2 m_{0} / \hbar^{2}\right)^{1 / 2}\left(\chi-E_{z}\right)^{1 / 2} s,
$$

which is rigorously correct only for a square barrier. If we define the cumulative current density as a function of energy according to

$$
j\left(E_{z}, s\right)=e \int_{0}^{E_{z}} d E_{z}^{\prime} D\left(E_{z}^{\prime}, s\right)\left[N_{0}\left(E_{z}^{\prime}\right)-N_{0}\left(E_{z}^{\prime}-q V\right)\right],
$$

the differential current density per unit energy (at energy $\left.E_{z}\right)$ is then

$$
\partial j\left(E_{z}, s\right) / \partial E_{z}=e D^{\mathrm{vac}}\left(E_{z}, s\right)\left[N_{0}\left(E_{z}\right)-N_{0}\left(E_{z}-q V\right)\right] .
$$

Assuming Boltzmann statistics, the condition for an extremum in this quantity is simply

$$
-(1 / k T)+\partial \ln D^{\operatorname{vac}}\left(E_{z}, s\right) /\left.\partial E_{z}\right|_{E_{z}=E_{m}}=0
$$

When appropriately generalized, this condition gives Eq. (30) and is relevant to the discussion following (32). In the present case, however, the extremum in (29) turns out to be a local minimum, and a maximum is instead found at the end point $E_{m}=0$. If the differential current density at this maximum is used to characterize the integrated tunneling current, Eq. (2) yields $\Phi_{A}=\chi$ for the apparent 
barrier height.

The next situation that is straightforward to analyze arises when we consider the effect of semiconductor space charge and ignore the vacuum barrier. This corresponds to the problem of tunneling through an ideal Schottky barrier structure. In this case, a WKB analysis reveals that the peak of the differential current distribution occurs at an energy $y^{31,32}$

$$
E_{m} / V_{d}=\left\{\cosh \left[q\left(\hbar^{2} N_{d} / 4 m_{z} \epsilon_{s}\right)^{1 / 2} / k T\right]\right\}^{-2} .
$$

In the limit of low doping and long depletion widths, this ratio approaches unity, so that the differential current density is proportional to $e^{-V_{d} / k T}$ and one recovers the thermionic emission picture of current transport across a Schottky barrier. ${ }^{30}$

The combined effect of the semiconductor space charge and a vacuum barrier is more difficult to follow through completely, but the qualitative features are apparent in at least one important limit. As noted in connection with Eq. (21), the semiconductor surface potential is a function of the vacuum gap width $s$. If one considers the physical1y realistic circumstance of $s / s_{0}<<1$, which corresponds to electrode separations that are small compared to the sample depletion width, the energy distribution will be almost completely determined by the semiconductor space charge. A specific example, based on the more accurate calculations described in Sec. II, is presented in Fig. 6, where we see that $E_{m} / V_{d}$ is nearly one, independent of $s$. Thus, for low doping, current transport is once again well described by a thermionic emission picture, and one finds from $\mathrm{Eq}$. (2) that $^{22}$

$$
\Phi_{A}^{1 / 2}=\chi^{1 / 2}+(1 / A)(q / k T)\left[\partial V_{d} / \partial s\right]
$$

Since the surface potential decreases with increasing sep-

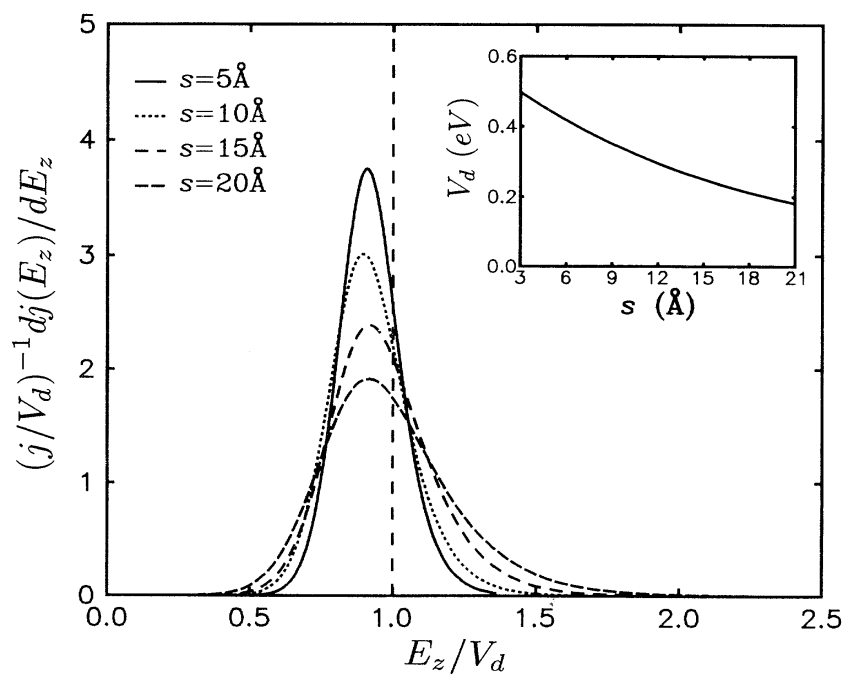

FIG. 6. Differential tunneling current density distribution versus normal energy $E_{z}$ (scaled to the semiconductor surface potential) as a function of electrode separation, neglecting image effects, for $V=-100 \mathrm{mV}$ and $N_{d}=5 \times 10^{17} \mathrm{~cm}^{-3}$. Inset: tip-induced semiconductor surface potential versus separation at the same fixed bias. aration, $s$, the second term in (31) is opposite in sign to the vacuum barrier contribution. The distance dependence of $V_{d}$ then has a profound influence on the apparent barrier height through its effect on the tunneling energy distribution.

Finally, we turn to the question of image forces and consider the case where we can neglect tip-induced band bending. A key issue is the extent to which image effects in the semiconductor interior perturb the energy distribution of tunneling electrons relative to that characteristic of the vacuum barrier alone. Ignoring image corrections in the vacuum region for simplicity, the extremum condition of Eq. (29) now becomes

$$
\begin{aligned}
-(1 / k T)+ & \partial \ln D^{\operatorname{vac}}\left(E_{z}, s\right) /\left.\partial E_{z}\right|_{E_{z}=E_{m}} \\
& +\partial \ln D^{\text {semicond }}\left(E_{z}, s\right) /\left.\partial E_{z}\right|_{E_{z}=E_{m}}=0,
\end{aligned}
$$

which includes a contribution from the image-induced barrier in the semiconductor. A useful estimate of the last term in (32) is provided by considering the limit of infinite separation between tip and sample. The semiconductor barrier is then a screened Coulomb potential due to a single image charge, as appropriate for the free semiconductor-vacuum interface. Since the proximity of the metal counterelectrode creates multiple image charges whose net effect limits the range of the semiconductor barrier, we may reasonably expect this approximation to provide an upper bound to the image-induced perturbation of the tunneling energy distribution. Employing the well-known expression for the energydependent WKB transmission coefficient through a Coulomb barrier, ${ }^{33}$ the relative significance of semiconductor and vacuum terms may be assessed via the dimensionless ratio

$$
\left[\beta\left(\frac{1-\beta}{1+\beta}\right)\left[\frac{m^{*}}{m_{0}}\right]^{1 / 2}\right]\left(\frac{\pi}{2}\right)\left(\frac{e^{2}}{16 \pi \epsilon_{0} \chi s}\right) .
$$

For typical values of the electron affinity in semiconductors, the last term in parenthesis is of order $(1 \AA / s)$, while the maximum possible value for the remaining parameters is 0.26. Evaluation for the specific case of $\mathrm{Si}(111)$ gives $0.05(1 \AA / s)$ for this ratio. Thus, at gap separations of order $10 \AA$ or more, the corrections to the maximum of the energy distribution are expected to be small for any material, and certainly negligible for silicon. We can inquire further, under the presumption that the semiconductor barrier will not be negligible under all circumstances, what may at most be expected for the shift in the location of the energy maximum. As an extreme circumstance, we retain the screened Coulomb potential in the semiconductor, while completely ignoring the vacuum barrier, and find

$$
\begin{aligned}
{\left[\frac{E_{m}}{k T}\right]^{3 / 2}=} & {\left[\beta\left[\frac{1-\beta}{1+\beta}\right]\left[\frac{m^{*}}{m_{0}}\right]^{1 / 2}\right] } \\
& \times\left[\left(\frac{\pi}{2}\right]\left[\frac{2 m_{0}}{\hbar^{2}}\right]^{1 / 2}\left[\frac{e^{2}}{16 \pi \epsilon_{0} k T}\right]\right] .
\end{aligned}
$$

For $\mathrm{Si}(111)$ at room temperature, $\left(E_{m} / k T\right)=0.76$, 
whereas for optimum parameters it will be no more than a factor of 3 greater.

The above considerations suggest therefore, that, relative to $\chi$, a zero-energy WKB analysis is an appropriate starting point for discussing how image forces influence the apparent barrier height in this system. That supposition is supported by numerical results obtained from the detailed formalism presented in Sec. II. In particular, Fig. 7 displays the differential current density per unit energy, as a function of gap spacing, calculated for the semiclassical multiple-image interaction using the one electron potential of Fig. 2(a). As one would expect, these distributions show resonant transmission for electron energies very near the conduction-band minimum, due to the shallow, long-range attractive portion of the image potential in the semiconductor interior, and, furthermore, they peak just below $\left(E_{z} / k T\right)=1$, as inferred above. Of particular note in Fig. 7 is that, in marked contrast to the situation for tip induced band bending (Fig. 6), the energy distribution for tunneling electrons appears to be only weakly dependent on $s$ (at least when the semiconductor barrier is of finite range), a relevant point outside the scope of our arguments thus far.

As a consequence of the scaling property (5) for the classical image potential in a semiconductor, the zeroenergy WKB transition coefficient through this barrier may be parametrized in terms of the dimensionless integral

$$
I(\beta)=\int_{\xi_{0}(\beta)}^{0}\left[U_{s}(\beta, \xi)\right]^{1 / 2} d \xi .
$$

It follows that the contribution of the semiconductor to the apparent barrier height at $E_{z}=0$ is given by

$$
\begin{aligned}
& \frac{\partial \ln D^{\text {semicond }}\left(E_{z}=0, s\right)}{\partial s} \\
& =I(\beta)\left[\frac{m^{*}}{m_{0}}\right]^{1 / 2}\left[\frac{2 m_{0}}{\hbar^{2}}\right]^{1 / 2}\left(\frac{e^{2}}{8 \pi \epsilon_{0} s}\right)^{1 / 2} .
\end{aligned}
$$

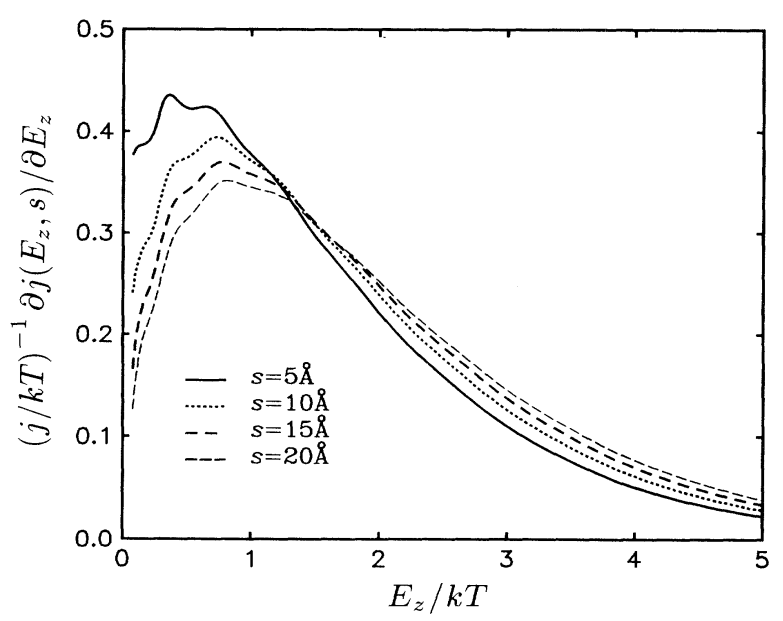

FIG. 7. Differential tunneling current density distribution versus normal energy $E_{z}$ (scaled to $k T$ ) as a function of electrode separation, including the (doping-independent) semiclassical image potential, but neglecting tip-induced band bending $\left(V=V_{\mathrm{FB}}\right)$
In relation to the vacuum barrier then, this represents a correction of order

$$
\begin{aligned}
& \left.\frac{\partial \ln D^{\text {semicond }}\left(E_{z}=0, s\right) / \partial s}{\partial \ln D^{\operatorname{vac}}\left(E_{z}=\right.}=0, s\right) / \partial s \\
& =I(\beta)\left(\frac{m^{*}}{m_{0}}\right)^{1 / 2}\left[\frac{e^{2}}{32 \pi \epsilon_{0} \chi s}\right]^{1 / 2} .
\end{aligned}
$$

Using our estimate above, the last term in parentheses is roughly $(1 \AA / 2 s)^{1 / 2}$. Now $I(\beta)$ has a maximum value of 0.51 at $\beta=1$ (an MVM junction), and decreases smoothly toward zero in the limit that $\beta$ vanishes. For any reasonable choice of the semiconductor dielectric constant, however, $I(\beta)$ exceeds 0.4 . Thus, in general, our correction term can at most be of order $0.3(1 \AA / s)^{1 / 2}$, and for $\mathrm{Si}(111)$ is only a factor of 2 smaller. Over a physically reasonable range of gap spacings from 10 to $1 \AA$, for example, the effect of the semiconductor image potential, in this approximation, will be to increase the apparent barrier height between $5 \%$ and $15 \%$.

Because of our reliance on a zero-energy analysis, the above estimate presents an upper limit to the magnitude of the effect one may reasonably expect. Indeed, our numerical calculations for $\mathrm{Si}(111)$ show that the inclusion of image forces produces a small net decrease in the apparent barrier height due to a reduction of the vacuum barrier, which is fully consistent with the results for MVM tunneling.

One may pause to consider at this point whether the situation will be fundamentally altered if we replace the far metal electrode with a second semiconductor whose polarizability is less than or equal to that of the first. In this case, the integral (35) diverges (with $\left|\xi_{0}\right| \rightarrow \infty$ ), since there is no longer any change in sign for $U_{s}\left(\beta, \beta^{\prime}, \xi\right)$ and the image-induced barrier is long range. Reflecting on (34), however, we are reminded that this barrier will be probed not at $E_{z}=0$, but rather at $E_{z} \approx k T$. The effective range of the image interaction at this energy is then of order

$$
z_{k T}=\beta\left(\frac{1-\beta}{1+\beta}\right)\left(\frac{e^{2}}{16 \pi \epsilon_{0} k T}\right),
$$

which, at $T=300 \mathrm{~K}$, is less than $25 \AA$ for optimum $\beta$. Thus, there is nothing in our arguments which leads us to suspect circumstances will be dramatically different for semiconductor-vacuum-semiconductor tunneling, unless one can arrange to conduct the experiments at low temperatures.

\section{SUMMARY AND CONCLUSIONS}

We have investigated the influence of the image potential on tunneling through a planar metal-vacuumsemiconductor junction. This problem differs qualitatively from the metal-vacuum-metal junction because there is now an image potential in the semiconductor interior as well as in the vacuum region. The classical multipleimage expression for an MVS system has been extended, using a Thomas-Fermi-like dielectric function in the semiconductor together with the usual Thomas-Fermi 
theory for the metal, to provide expressions which are everywhere finite. The resulting semiclassical image potentials in semiconductor, vacuum, and metal must still be smoothly joined, however, by an interpolation through the interfaces. Using these potentials, the tunneling current at finite temperature was calculated as a function of separation for the particular case of a n-type hydrogen-passivated $\mathrm{Si}(111)-\mathrm{Au}$ system, and an apparent barrier height $\Phi_{A}$ then extracted to simulate the results of STM modulation experiments.

To obtain a more general understanding of these detailed calculations, qualitative arguments based on a WKB picture have also been presented. If the image potential is neglected, $\Phi_{A}$ is well described by terms involving, first, the semiconductor's electron affinity, and, second, the effect of tip induced band bending. When the contribution of the image potential within the semiconductor is separately considered, a WKB estimate indicates it will perturb the apparent barrier height by at most $10 \%$ at reasonable tip-sample distances. This crude estimate helps one understand the principal result of the detailed calculations presented in Figs. 3 and 4: Although the tunneling barrier for an MVS junction differs from that for an MVM junction, the image potential in the semiconductor makes only a modest contribution to the apparent barrier height. Furthermore, as our calculations reveal, the dominant effect is a slight lowering of $\Phi_{A}$, due to the attractive image potential in the vacuum region in conformity with expectations based on MVM tunneling, rather than any increase in $\Phi_{A}$ from the repulsive image potential within the semiconductor.

One may have thought that the semiconductor's internal image potential, being long range, would have a substantial effect on carriers originating from the bulk conduction-band edge, and thus influence not only tunneling currents, but the apparent barrier height as well.

${ }^{1}$ J. G. Simmons, J. Appl. Phys. 34, 2581 (1963).

${ }^{2}$ J. G. Simmons, in Tunneling Phenomena in Solids, edited by E. Burstein and S. Lundqvist (Plenum, New York, 1969).

${ }^{3}$ N. M. Miskovsky et al., Appl. Phys. A 27, 139 (1982).

${ }^{4}$ N. D. Lang and W. Kohn, Phys. Rev. B 7, 3541 (1973).

${ }^{5}$ N. D. Lang, Solid State Phys. 28, 225 (1973).

${ }^{6} \mathrm{G}$. Binnig et al., Phys. Rev. B 30, 4816 (1984).

${ }^{7}$ N. D. Lang, Phys. Rev. B 37, 10395 (1988).

${ }^{8}$ J. H. Coombs, M. E. Welland, and J. B. Pethica, Surf. Sci. 198, L353 (1988).

${ }^{9}$ J. Bono and R. H. Good, Surf. Sci. 151, 543 (1985).

10J. Mahanty and M. T. Michalewicz, J. Phys. C 19, 5005 (1986).

${ }^{11}$ H. Morawitz et al., Surf. Sci. 180, 333 (1987).

${ }^{12}$ A. A. Lucas et al., Phys. Rev. B 37, 10708 (1988).

${ }^{13}$ S. Ossicini and M. Bertoni, Phys. Rev. B 35, 848 (1987).

${ }^{14}$ R. Garcia, Phys. Rev. B 42, 5476 (1990).

${ }^{15}$ B. N. J. Persson and A. Baratoff, Phys. Rev. B 38, 9616 (1988).

16J. Bono and R. H. Good, Surf. Sci. 175, 415 (1986).

${ }^{17}$ M. Kleefstra and G. C. Herman, J. Appl. Phys. 51, 4923 (1980).

${ }^{18}$ A. Tugulea and D. Dascalu, J. Appl. Phys. 56, 2823 (1984).

${ }^{19}$ R. Browning, M. A. Sobolewski, and C. R. Helms, Phys. Rev. B 38, 13407 (1988).

${ }^{20}$ J. C. Inkson, J. Phys. C 6, 1350 (1973).
Our calculations together with the WKB analysis, indicate otherwise. Three qualitative arguments account for this observation: First, in the case of flatbands, the potential due to polarization charge at the vacuum-metal interface competes with that of the semiconductorvacuum interface to produce a barrier in the semiconductor interior which is short range; second, for the more general case which includes a semiconductor diffusion potential in either depletion or weak inversion, the overwhelming influence on the carrier distribution arises from the diffusion potential, and not the image potential. In the case this diffusion potential is tip induced, we have shown explicitly that $\Phi_{A}$ is almost completely dominated by the distance dependence of field penetration in the semiconductor, and that image effects are of relatively minor importance; finally, for flatband conditions, most of the tunneling current is provided by electrons whose energy is of order $k T$ above the band edge. For these electrons, the image-induced barrier is effectively short range at room temperature, even if one neglects the influence of polarization charge at the metal tip. The same argument applies, in principle, to the semiconductor-vacuum-semiconductor junction, but whether or not the image potential will have a significant influence in this system at low temperatures remains an open question.

\section{ACKNOWLEDGMENTS}

We gratefully acknowledge J. A. Kramar for providing part of the computer codes on which these calculations were based. This work was supported by the Office of Naval Research under Grants No. N00014-89-J-3029 and No. N00014-91-J-1126, and by the Robert A. Welch Foundation.
${ }^{21}$ Z.-H. Huang, M. Weimer, and R. E. Allen, J. Vac. Sci. Technol. B 9, 2399 (1991).

${ }^{22}$ M. Weimer, J. Kramar, and J. D. Baldeschwieler, Phys. Rev. B 39, 5572 (1989).

${ }^{23}$ J. C. Inkson, J. Phys. C 4, 591 (1971).

${ }^{24}$ D. M. Newns, J. Chem. Phys. 50, 4572 (1969).

${ }^{25}$ N. W. Ashcroft and N. D. Mermin, Solid State Physics (Holt, New York, 1976).

26J. P. Walter and M. L. Cohen, Phys. Rev. B 2, 1821 (1970).

${ }^{27}$ S. M. Sze, Physics of Semiconductor Devices (Wiley, New York, 1981).

${ }^{28}$ C. B. Duke, in Tunneling in Solids, edited by F. Seitz, D. Turnbull, and H. Ehrenreich, Solid State Physics (Academic, New York, 1969), Suppl. 10.

${ }^{29}$ J. A. Kramar, Ph.D. thesis, California Institute of Technology (1990).

${ }^{30}$ E. H. Rhoderick and R. H. Williams, Metal-Semiconductor Contacts, 2nd ed. (Oxford University Press, New York, 1988).

${ }^{31}$ F. A. Padovani and R. Stratton, Solid-State Electron. 9, 695 (1966).

${ }^{32}$ C. R. Crowell and V. L. Rideout, Solid-State Electron. 12, 89 (1969).

${ }^{33}$ L. D. Landau and E. M. Lifshitz, Quantum Mechanics (Nonrelativistic Theory), 3rd. ed. (Pergamon, New York, 1977). 